\title{
REDESIGN OF THE EUROPEAN ROUTE NETWORK FOR SECTOR-LESS
}

\author{
Thomas Rivière, CENA-Eurocontrol, Toulouse, France
}

\begin{abstract}
The increase in air traffic and the limited capacity of air traffic control services force us to think of a new way in which to control aircraft. An innovative ATM concept, called Sector-Less Air Traffic Management, has been defined by the Eurocontrol Experimental Centre.
\end{abstract}

In this concept the role of the controller is radically different from the current one: instead of having controllers controlling a sector, controllers will be responsible for a limited number of aircraft, from departure to arrival in terminal areas. Within this framework, the European air space will need to be redesigned in order to minimise the number of conflicts and to give an aeroplane the shortest route possible by providing a new route network.

The approach proposed here is, starting from scratch, to provide a very simple route network and to improve it by using optimisation techniques. The generated route network will be evaluated in terms of length of trajectories weighted by the amount of aeroplanes using them and compared with the direct route network and the current one.

Using this tool and a fast time air traffic simulator, The Sector-Less concept will be evaluated in terms of capacity and delay due to conflicts. This will also provide the route density of the network necessary for this concept.

\section{Introduction}

The increase in air traffic raises a major concern. How does one simultaneously accommodate increasing numbers of aircraft into an already saturated airspace, whilst maintaining safety at at least current levels, and simultaneously improving the efficiency of Air Traffic Services by reducing delays? An innovative ATM concept, called Sector-Less Air Traffic Management, defined by [2] at the ATM conference, 2001 tries to answer this question.

In this concept the role of the controller is radically different from the actual one: instead of having two controllers controlling one sector containing $n$ aircraft, one controller will be responsible for a limited number of $m$ aircraft, from departure to arrival terminal areas (TMA).

According to [2] the mean number of operations handled by one controller per year does not exceed 650. Mathematically speaking, instead of managing a number of aircraft flying across a number of sectors, requiring the attention of a number of controllers responsible for those sectors, it is not unrealistic to assume that the same number of controllers can handle such numbers of aircraft individually from departure to arrival.

The aim of this paper is to generate a route network which will fit into the Sector-Less concept and provide an aeroplane with the shortest route possible. This problem will be approached by generating a suitable simple route network for Sector-Less and improved by using optimisation techniques such as simulated annealing algorithm.

After having roughly explained the Sector-Less concept in the second part, this paper will show how, within this framework, a route network is generated and optimised.

\section{The Sector-Less concept}

\section{The Concept}

This concept has been devised by taking into account one major problem in the current ATC world: traditionally the traffic increase has been accommodated by subdividing highly loaded sectors but nowadays many sectors have become too small to be divided. [2] think that now sectors are therefore a constraint to the increase of air traffic and that there is obviously a need to explore new practices that could break away from this major constraint.

The paradigm that Sector-Less investigates is the trajectory-based individual control as opposed to the airspace-based sector control currently used. The ultimate unknown which this project tries to clarify is whether or not in the future we could remove sectors, as well as their associated 
constraints, in order to respond to the capacity impasse.

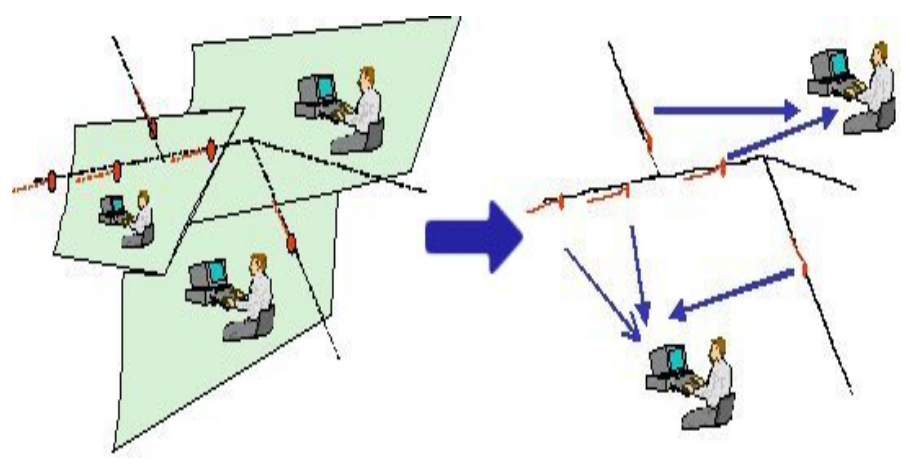

Figure 1. Basic Sector-Less Concept

\section{The Airspace Design}

According to [2] the generation process of a route network for their concept can be divided into two sub-problems:

- a Trunk Route Network (TRN) in which there will be specific spacing techniques performed whilst avoiding flight level changes and vectoring ;

- a Secondary Route Network (SRN) which will link the TRN to every origin and destination TMA.

The main airspace design rules of the SectorLess concept are composed of the one which separates flows going in opposite directions and the one which handles the crossing section between 2 routes. As shown in Figure 3, the crossing rule works more or less like a roundabout.

\section{Flows Separation}

The airspace design has been defined by [4] and [5] in an ATC concept called Super-Sector. This concept has been devised in order to create a transition between the actual ATC system and the Sector-Less one. Super-Sector is a mixed ATFM concept : it has large sectors (which could be as big as a country) and every of them contain its own TRN and SRN. Thus, aeroplanes flying into these sectors will be following the local route network yet be controlled locally and not from departure to arrival. The airspace designed by [4] consists of a 3D tube structure (tubular airspace) and has 3 main characteristics:
- Trunk-Highway (Trunk Route Network)

- Parallel route to support offset capabilities

- Flight Level Allocation System (FLAS)

Some of the rules therefore defined in SuperSector must be used in Sector-Less. As the concept implies a simplification of the route network, it is possible to think of larger routes than the current one by employing closed parallel tracks. This will allow fast aircraft to overtake slower ones. We can also imagine using tracks to change flight levels without causing any problems to other flights.

As shown in Figure 2, which represents a crossroad of 2 orthogonal flows, every route is doubled in order to avoid mixing traffic going in opposite parallel directions. In addition to that, every route is comprised of 3 tracks: a main track and two secondary ones, respectively on each side of the main track, used for manoeuvring techniques. Moreover this figure shows two flows going in orthogonal directions and the crossing techniques this concept requires. These techniques will be presented in the next part of this paper.

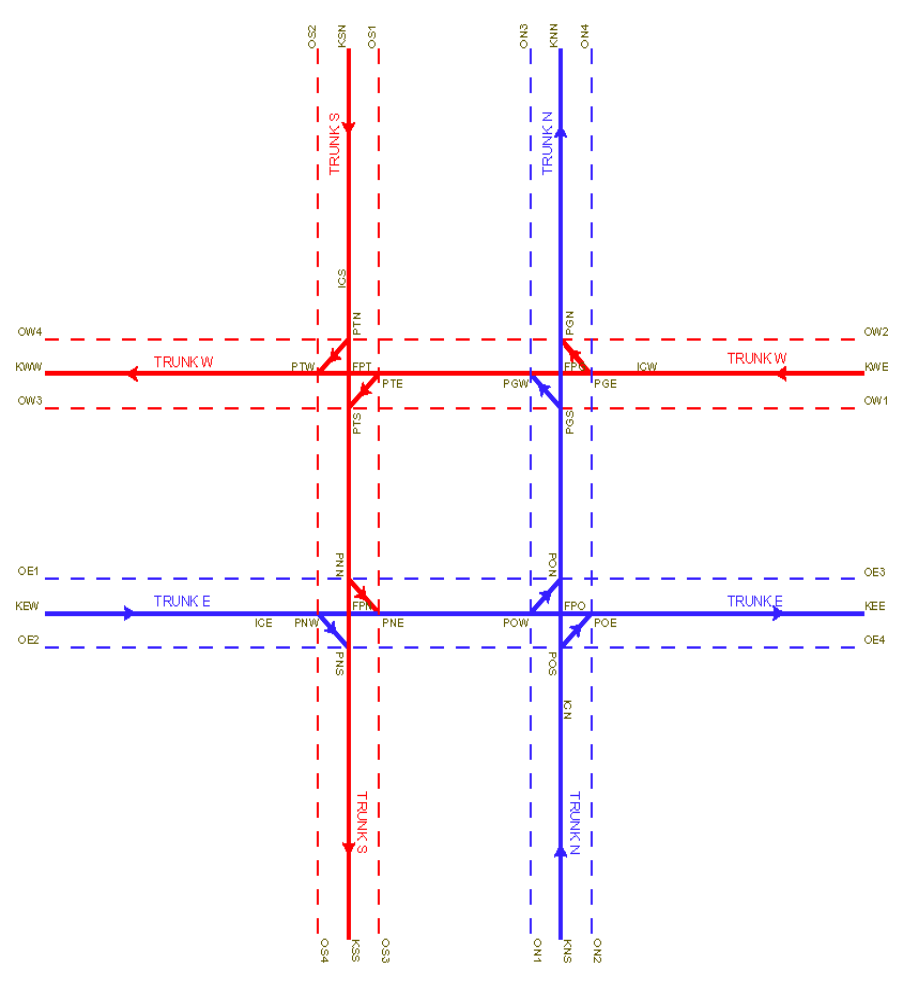

Figure 2. Super-Sector Flows Separation System 
Finally, the rules taken into account for the flows separation are that:

- flows going in opposite directions use parallel routes. Unlike [5] in the SuperSector concept, aircraft going in opposite parallel directions are allowed to use the same flight level but, consequently, parallel routes must be far enough apart in order to respect the safety distance;

- one route can be divided into several parallel tracks going in the same direction in order to allow faster aeroplanes to overtake slower ones;

- 2 crossing routes must be on a different flight level. One can therefore imagine that aircraft taking a directional route East-West bound have an odd flight level so aircraft going in a South-North direction have an even flight level.

As opposed to the Super-Sector concept where a crossing point has to be over an airport, it is assumed in the following sections that they can be placed wherever they need to be in order for the route network to be optimised ${ }^{1}$.

\section{Crossing Points}

In order to make this flows separation rule available, the Sector-Less and the Super-Sector concepts define another important rule on how to handle the crossing section of 2 flows.

As shown in Figure 3, a crossing point in the Sector-Less concept works more or less like a roundabout, but uses 3 dimensions.

As mentioned in the previous section, 2 crossing routes must use different flight levels. An aeroplane which is crossing another route has to respect the following rules (see Figure 4):

- any aeroplane passing through without changing direction must stay stable on its flight level;

- any aeroplane turning right must not cross the route but must change flight level (be that up or down) to reach the flight level of their new trunk route ;

\footnotetext{
${ }^{1}$ If some of the crossings are fixed, the optimisation process does not make sense.
}

- any aeroplane turning left must cross the route, turn at the opposite corner and change flight level.

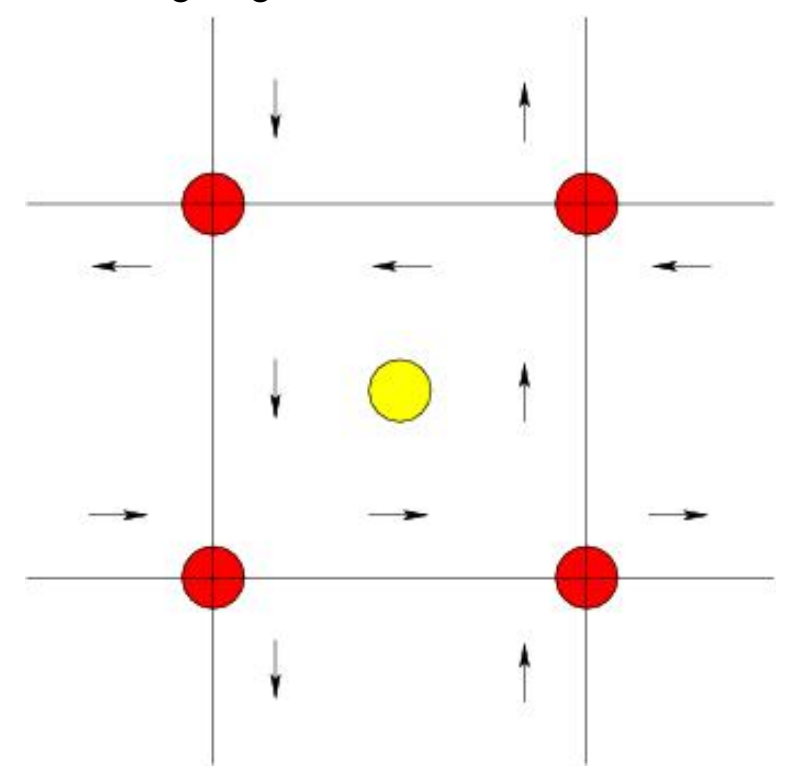

Figure 3. The Square Crossing

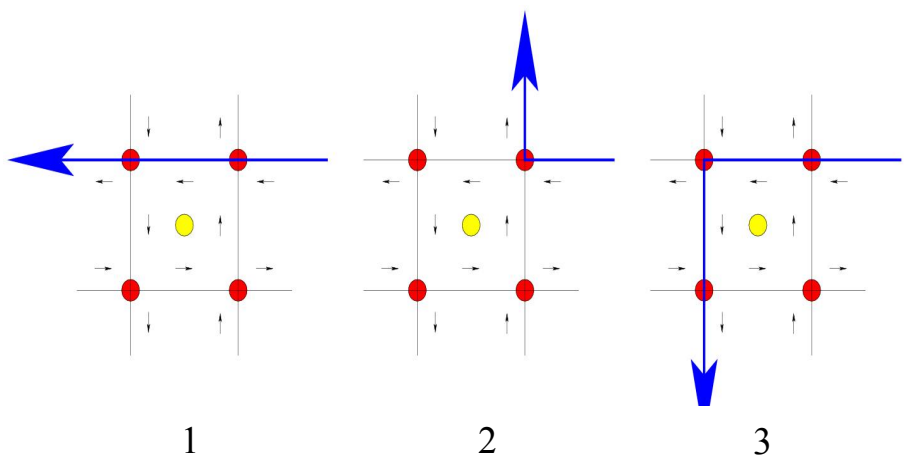

Figure 4. The Turning Process

According to the rules defined above, it is comprehensible that 2 main criteria are going to lead the research: these seek to find the shortest path possible and to minimise the number of turning points.

\section{The Trunk Route Generation}

As the Sector-Less concept is something radically different from the current one, a new route network will be generated for it starting from scratch rather than being an adaptation of the current one. Except for the basic Air Traffic Management rules, no technical configuration data 
concerning the route network has been defined by [2] or [5].

The prime concern of this research is to generate the best Trunk Route Network possible. The secondary route network has not yet been taken into account so the only way to reach or leave the trunk route network is by using direct routes.

\section{The Initial TRN}

The first route network generated is a very simple square grid covering Europe (see Figure 5). Every parameter has been decided arbitrarily; future work will be to test different values for each one and find the best of these.

The initial TRN has the following characteristics:

- a square 4000 kilometres long;

- two neighbouring crossing points separated by $240 \mathrm{~km}$ making 256 crossing points.

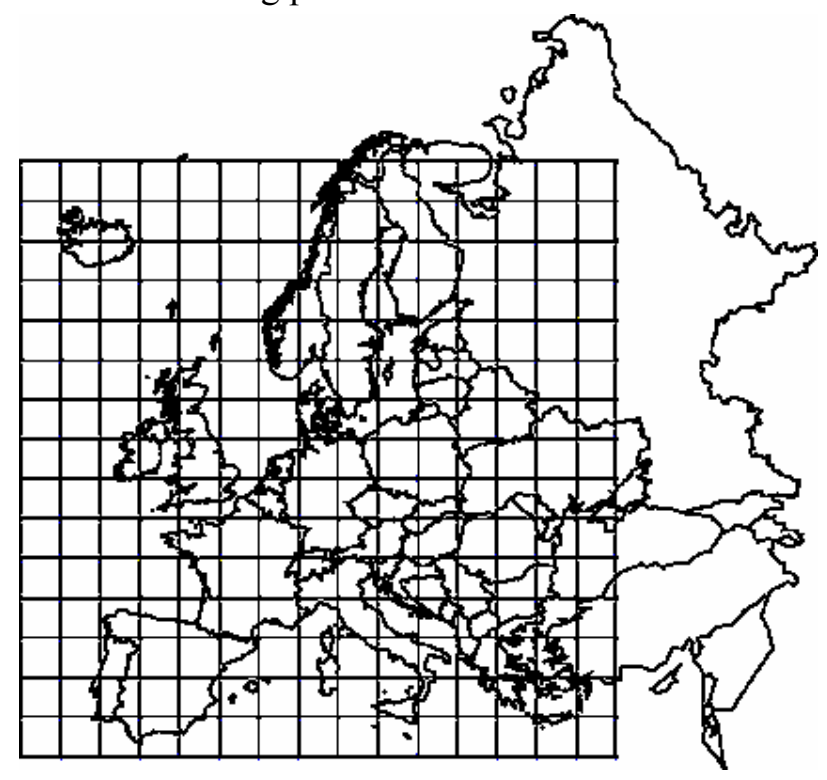

Figure 5. The Initial TRN

\section{Grid Bending}

The aim of this work is to optimise the basic route network defined previously by bending the grid according to one essential criterion: the average global extension of trajectories in comparison with the length of direct routes.
In the case of the initial TRN, the criterion is worth $32 \%$ which means that a trajectory is, on average, $32 \%$ longer than the direct route.

\section{Simulated Annealing and Shortest Path Algorithms}

The route network is optimised by using an algorithm based on a simulated annealing algorithm [6]. Thus, the algorithm:

- chooses 1 point randomly and moves it in a random direction;

- evaluates the criterion;

- rejects or accepts the movement.

The acceptance of a movement is performed:

- if the criterion is improved;

- if the criterion is deprecated. The movement is accepted only under certain conditions that will be discussed later.

The optimisation criterion is based on a FloydWarshall shortest path algorithm [3]. It gives the shortest path between every pair of points on the grid. Knowing every air flow possible ${ }^{2}$ and the number of aeroplanes using $\mathrm{it}^{3}$, the average global extension of trajectories (which is the sum of the differences between the length of a trajectory and the corresponding direct route, weighed by the number of aeroplanes using this route) can easily be computed.

Consequently the criteria favours the flows with a high number of flights per day ${ }^{4}$.

\section{Limitations}

It is worthwhile noting that some limitations have to be added in order to provide a route network which is potentially valid in reality:

- the distance between 2 crossing points cannot be smaller than 100 kilometres in

\footnotetext{
${ }^{2}$ A departure-arrival pair is considered in this paper as a flow. ${ }^{3}$ Arbitrarily the data of June the 21 st which is one of the busiest day of the year 2002 with 10738 flows has been chosen. ${ }^{4}$ In Europe the main flows are Madrid-Barcelona (over 70 airplanes a day in each direction), Milan-Rome (about 45 airplanes a day), Paris-London (35) or Paris-Toulouse (35).
} 
order for an aircraft to spend more time on a main trunk than in a crossing area;

- a shortest path cannot include an angle smaller than 90 degrees. This is in order to make turning points feasible. As the criteria favours the main flows, some flows (which are mainly orthogonal to these major flows) with only a few aeroplanes a day may have awkward trajectories (see Figure 6) with turning angles impossible to manage in reality.

The last limitation tends to smooth trajectories.

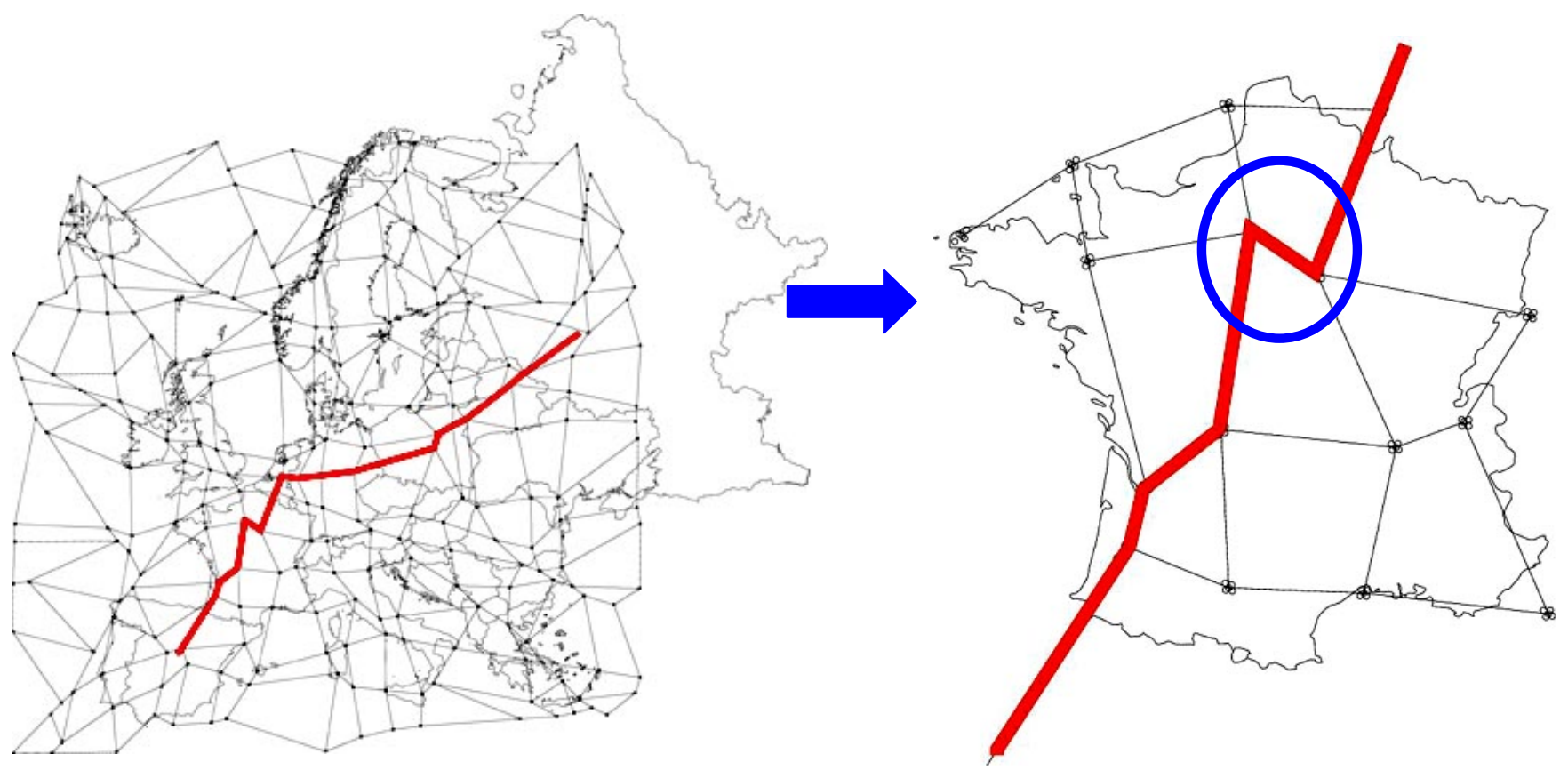

Figure 6. Moscow-Madrid Rejected Trajectory

\section{Various Heuristics}

The main problem of this simple algorithm is the large width of the search space, the data size used by the shortest path algorithm and the number of variables of the optimisation criterion. In order to find a better solution and/or to find a good solution more quickly different heuristics have been tried:

\section{- Acceptance and rejection of a bad movement:}

As in most simulated annealing algorithm, we use a decreasing "temperature" and a probability of acceptance of a solution equal to $\exp (-$ cost/temp). With this solution, bad movements are often accepted at the beginning of the search and very rarely at the end of it. However we have still not found a good decreasing rule for the temperature which could lead to a more efficient algorithm.
Finally the temperature has not been taken into account. Thus, a bad movement is accepted if, after a certain number of steps, no improvement of the criterion has been made. This method is not as good as the previous one but gives roughly the same quality of solution and is easier to use.

\section{- Floyd every $\boldsymbol{n}$ steps:}

The Floyd-Warshall algorithm can only be employed after a certain number of steps and not for every single one. This algorithm therefore recalculates every shortest path between each point on the grid. Moving one point, however, does not change every single path.

Consequently, in order to reduce the calculation time, several points were moved before the recalculation of every shortest path but this 
technique seems to have degraded the quality of the solution found.

\section{- Choosing the point to move:}

Rather than randomly selecting the point to move, it was purposefully chosen. As the optimisation criterion is the lengthening of trajectories, it was decided that points of the longest flows should be moved but no improvement has yet been observed using this method.

\section{Best TRN so far}

The best trunk route network obtained so far (Figure 7) has been deducted from the initial one presented before using the basic algorithm described previously.

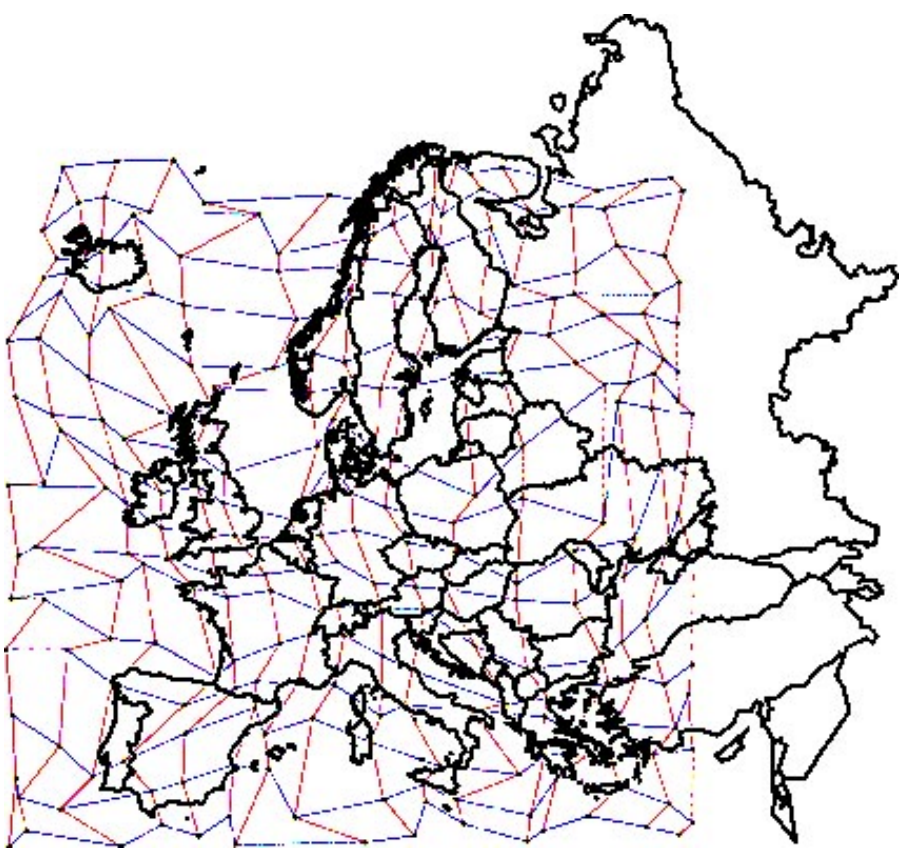

Figure 7. The Best TRN So Far

The average global extension of trajectories in comparison with the length of direct routes is worth $16 \%$ in this case.

Figure 8 represents the trajectory of an aeroplane going from Reykjavik, Iceland (BIKF) to Palma, Mallorca Island, Spain (LEPA) using this TRN.

\section{Conservation of the Airspace Design Rules}

Of course, the fact that the grid is bent has an influence on the airspace design rules defined previously. Even though the bending process does not change anything in the flows separation rule, the shape of the crossing section cannot remain square.

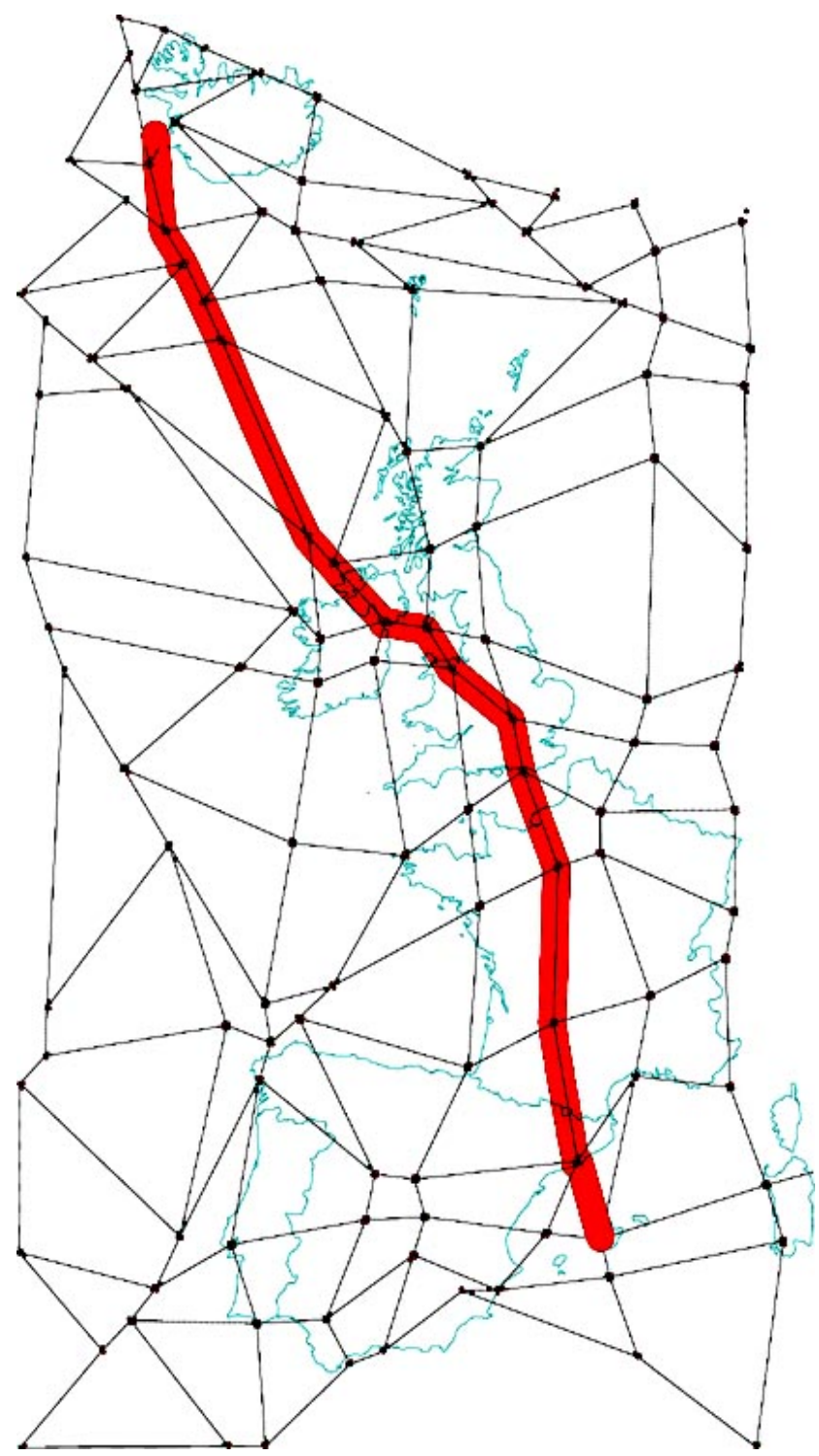

Figure 8. Example of Trajectory in the TRN

As presented in figure 9, every crossing section has to be adapted to its new shape. Its size and the relative position of its 4 turning points (with respect to its centre) change. The only parameter which does not change is the distance between 2 parallel routes. 


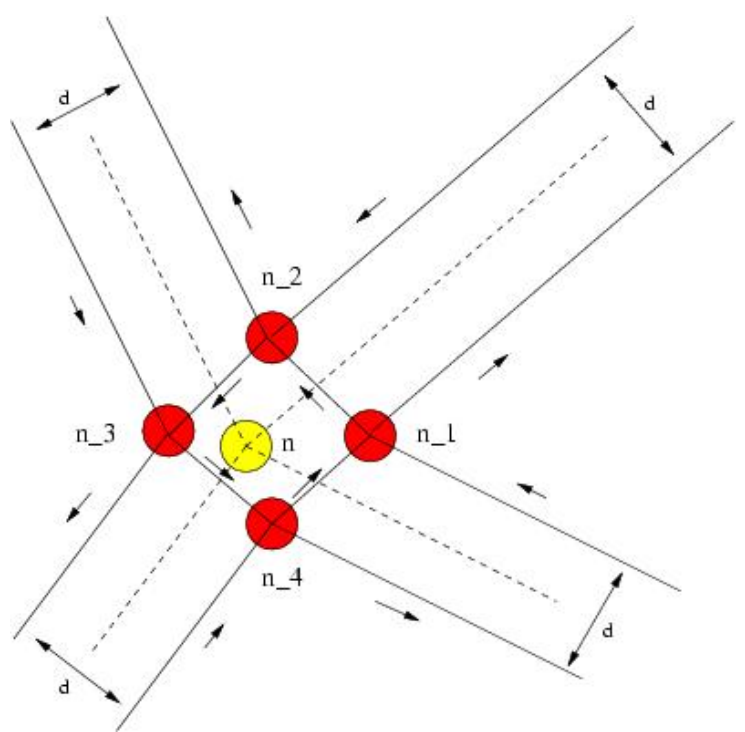

Figure 9. Adaptation of the Crossing Section

\section{First Evaluation}

The first comparison was made with the already existing route network. In using the same criterion, one will find that the average extension of the current network is between $7 \%$ and $11 \%{ }^{5}$. The difference could be considered important but one still has to keep in mind that the goal of this research is to find a simpler route network and, in fitting with the Sector-Less concept, to have a bigger capacity.

Several positive points can be seen on the generated TRN (see Figure 7):

- the crossing points tend to go where they are required : for example there are none above the North sea due to the fact that no aeroplanes need to change direction over this area;

- the crossing points tend to have a distribution which follows the main flows (because of the criterion) such as over France where there is a TRN segment on the London-Paris flow and another on the Toulouse-Marseille flow;

- the crossing points tend to group themselves together on the "exterior" of the grid (especially over Russia) which

\footnotetext{
${ }^{5}$ The value of the criterion depends on routes taken by aircraft because in the current route network, for an origin-destination flow, several routes are available
}

may mean that too many of them are being used.

On the other hand, the fact that the TRN segments tend to follow the main flows puts the traffic in one place. For example, rather than reaching Spain by flying over the ocean, the shortest path of the aeroplane in figure 9 , will be over western Europe and will take the same segments as several major flows (here London-Paris and Paris-Toulouse). This could potentially increase the number of conflicts.

\section{Conclusion and Further Work}

The first results are encouraging but more evaluations have to be made in order to be able to evaluate different route networks. For a further evaluation of a TRN, two points of view have to be taken into account:

- that of the user, in terms of extension of trajectories and of level allocation;

- that from the control point of view, in terms of regulations and conflicts. This could be done by simulating certain traffic days on the fast time simulator CATS (see [1]) using real data.

It could also be possible to evaluate the SectorLess concept in terms of capacity and conflicts.

Furthermore, some other optimisation algorithms such as genetic algorithm (see [7]) could be applied in order to generate the best TRN possible and better heuristics for the choice of the point to move could also be found. Finally, the number of turning points should be minimised.

\section{References}

[1]JM Alliot, JF Bosc, N Durand, and L Maugis, 1997, Cats: A complete air traffic simulator. 16th DASC.

[2] Vu Duong, Gilles Gawinowski, Jean-Pierre Nicolaon, and Darren Smith. Sector-Less Air Traffic Management, December 2001, In 4th USA/Europe Air Traffic Management R\&D Seminar, Santa Fe.

[3]R.W. Floyd, June 1962, ACM Algorithm 97: Shortest path. j-CACM, 5(6), pp. 345. 
[4]Gilles Gawinowski, Jean Nobel, Didier Dohy, Jean-Yves Grau, and Vu Duong, 2003, Bridging the predictive and adaptative issues in air traffic

management : the synchronous paradigm, In 22nd

Digital Avionics Systems Conference, Indianapolis.

[5]Gilles Gawinowski, Jean Nobel, Didier Dohy, Laurent Guichard, and Vu Duong, 2003,

Operational Concept For Super-Sector, In 5th

USA/Europe Air Traffic Management R\&D

Seminar, Budapest.
[6]P. J. M. van Laarhoven and E. H. L. Arts, 1987, Simulated annealing: theory and applications, Kluwer Academic Publisher.

[7]Jean-Marc Alliot and Thomas Schiex , 1994, Intelligence artificielle et informatique théorique, Cépaduès éditions.

\section{Email Address}

Thomas Rivière, riviere@recherche.enac.fr 